\title{
Editorial
}

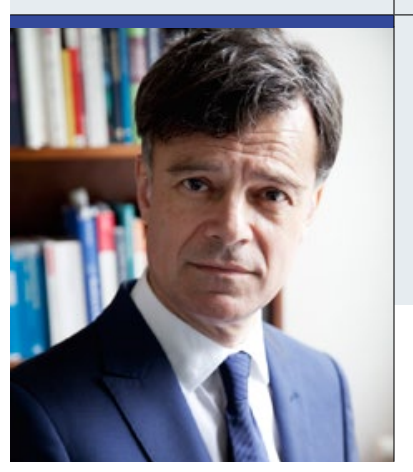

Medizin ist eine Disziplin, die ihre Leistung in der direkten

Interaktion vollzieht und ihre Qualität bemisst sich nach dem

Kriterium der gelingenden Interaktion im Hier und Jetzt.

Prof. Dr. med. Giovanni Maio, M.A. phil., Freiburg

Lehrstuhl für Medizinethik

\section{Die Kunst der Verknüpfung von Faktenwissen und Hermeneutik}

$\mathrm{n}$ einer auf hochtourige Betriebsamkeit umgestellten Medizin bleibt kein Raum mehr für ein vertieftes Nachdenken darüber, worin die Identität der Medizin besteht und was ihre Kernleistung ist. Stattdessen findet gegenwärtig eine sukzessive Umerziehung der Ärzte statt, die dazu angehalten werden, allein dem Formalisierbaren Beachtung zu schenken und alles andere als irrelevant anzusehen. Je mehr politisch gesteuert eine überbordende Kontrolle und damit eine Bürokratisierungsspirale über die Medizin verhängt wird, desto mehr wird die Aufmerksamkeit der Ärzte umgelenkt auf das Dokumentier- und Kontrollierbare. Die Orientierung an partikularen Parametern wirkt als Aufmerksamkeitsverzehrer, das Dokumentierbare zieht alle Aufmerksamkeit auf sich und lässt keinen Raum für das Nicht-Messbare. Diejenigen, die sich diesem Postulat widersetzen, geraten unweigerlich in die Defensive und sehen sich nicht nur dem Vorwurf der Ineffizienz, sondern dem Vorwurf der Beliebigkeit, ja gar der Unwissenschaftlichkeit ausgesetzt.

Wissenschaftlichkeit in der Medizin bedeutet aber gerade, dass die Heilberufe das Formalisierbare mit dem Lebensweltlichen zusammenführen müssen. Allein nach Zahlen wird man nicht helfen können, weil man allein nach Zahlen den kranken Menschen schlichtweg nicht verstehen kann. Die Medizin braucht eben beides; sie braucht Zahlen, sie braucht naturwissenschaftliche Daten, sie braucht Statistik und externe Evidenz. Aber mit dieser Evidenz allein wird sie ratlos bleiben, denn Aufgabe der Medizin ist ja nicht, Algorithmen umzusetzen. Ihre Aufgabe besteht darin, eine Antwort auf die Not des Patienten zu finden, und diese Antwort findet sich nicht auf dem Reißbrett, sondern sie muss kreativ erschlossen werden in der Begegnung mit dem Patienten.

Medizin ist eine Disziplin, die ihre Leistung in der direkten Interaktion vollzieht und ihre Qualität bemisst sich nach dem Kriterium der gelingenden In- teraktion im Hier und Jetzt: Ihre Qualität ist die Qualität der Beziehung. Daher braucht die Medizin mehr als Regeln, sie braucht Raum für das Entstehen einer Vertrauensbeziehung, sie braucht Atmosphären, sie braucht innere Ruhe und Freiheit, um sich scheuklappenfrei auf den einzelnen Patienten einzulassen.

Medizin hat immer damit zu tun, das richtige $\mathrm{Maß}$ zu finden. Das richtige Maß ist eben kein arithmetisches Maß, sondern eines, das erst gefunden werden kann durch rationale Analyse der Elemente, die den Einzelfall konstituieren. Die Könnerschaft der Heilberufe liegt darin, Komplexität angemessen zu bewältigen. Jeder Patient bringt sie mit sich, Komplexität seiner Vorgeschichte, seiner Lebensgeschichte, seiner momentanen Situation, seiner Perspektiven. Diese Komplexität zu erfassen, erfordert ein bestimmtes Wissen, das mit dem Begriff der ärztlichen Kunst erfasst war, das aber mit der heutigen Vorstellung einer industrialisierten Medizin sukzessive verlernt wird. Es ist nämlich ein interpretatives, kontextuelles, hermeneutisches Wissen. Wenn man dem Patienten gerecht werden will, muss man verständigungsorientiert vorgehen, um die Situation des Patienten zu verstehen und durch die Kommunikation in gemeinsamer Arbeit einen dem Patienten gerecht werdenden Weg zu finden.

Medizin zu betreiben bedeutet, über eine solche Wissensbasis zu verfügen, die es erlaubt, das theoretische Sachwissen so mit einem praktischen Handlungswissen zu verbinden, dass am Ende vermittels einer zu erlernenden praktischen Urteilskraft eine ärztliche Therapieempfehlung steht, die dann wissenschaftlich solide ist, wenn sie der Individualität des Patienten gerecht wird. Diese individuelle Therapie in verständigungsorientierter Weise herauszufinden, ist die anspruchsvolle Leistung der Heilberufe, die als unersetzbar anerkannt werden muss.

Prof. Dr. med. Giovanni Maio 